\title{
The Use of Sulfasalazine in Atrophie Blanche
}

\author{
Aditya K. Gupta, M.D., F.R.C.P.(C.), Michael T. Goldfarb, M.D., and John J. Voorhees, M.D.
}

\begin{abstract}
Atrophie blanche can be a chronic condition for which there is no satisfactory treatment. Two patients with atrophie blanche who had not responded to various therapeutic modalities were given a trial of sulfasalazine $1 \mathrm{~g}$ three times daily. The ulcers healed within 3 months in both cases. In view of these positive results, patients should be treated with sulfasalazine to determine the efficacy of this drug in atrophie blanche.
\end{abstract}

Atrophie blanche was first described by Milian in 1926. ${ }^{1}$ Others ${ }^{2,3}$ have preferred the term livedo vasculitis or segmental hyalinizing vasculitis for this condition. It is a chronic disorder that is more common in young and middle-aged women and usually affects the lower legs and ankles. Uncommonly, lesions may occur on the hands, face, and trunk. There is usually no underlying systemic disease. The salient pathologic findings include the occurrence of fibrinoid material in superficial blood vessels, decreased vascularity, ${ }^{4}$ and intimal proliferation as well as hyalinization of the blood vessel walls. ${ }^{2,3}$ However, there are no uniformly accepted clinical or histopathologic criteria for this condition. ${ }^{4,5}$ We report the successful use of sulfasalazine in two patients with atrophie blanche (livedo vasculitis), a condition in which none of the numerous reported treatments has been consistently effective. ${ }^{6-8}$

\section{Case 1}

- An 18-year-old white woman presented in August 1988 with a 3-year history of painful, multiple ulcerations of both her lower legs. For the previous 2 weeks, the pain had been worsening with a decreasing range of motion of the right ankle, necessitating the use of crutches. The patient was otherwise in excellent health and had no symptoms of collagen vascular or Raynaud disease. Past therapies included trials of pentoxifylline, nifedipine,

From the Department of Dermatology, University of Michigan Medical Center, Ann Arbor, Michigan.

Supported in part by the Babcock Endowment Fund.

Address correspondence to: John J. Voorhees, M.D., Department of Dermatology, University of Michigan Medical Center, Room 1910, Taubman Center, 1500 East Medical Center Drive, Ann Arbor, MI 48109-0314. ibuprofen, and naproxen, all of which had been of no therapeutic benefit.

On examination, there was erythema and edema of the lower aspects of both legs and ankles. Numerous purpuric macules and papules were noted with multiple punched-out irregularly shaped, tender ulcerations, $8-15 \mathrm{~mm}$ in diameter (Fig. 1A). There were also areas of stellate, ivory-colored, atrophic scars, some of which had a hyperpigmented border with telangiectasias. The disease affected the right leg to a greater extent than the left. The range of active motion of the right ankle was markedly reduced. She had normal peripheral pulses and no evidence of varicosities or stasis dermatitis.

Laboratory investigation, including CBC, SMA12, ANA, complement levels, cryoglobulins, serum protein electrophoresis, VDRL, and urinalysis were negative or within the normal range. An x-ray of the right ankle was normal. Histology was consistent with atrophie blanche and revealed intravascular fibrinoid deposits and prominent hyalinization of the middle and deep dermal vessel walls.

The patient was started on sulfasalazine $500 \mathrm{mg}$ tid for 3 days, followed by $1 \mathrm{~g}$ tid. The only topical treatment used by the patient consisted of the daily application of a dry dressing. At pretherapy, the patient was taking acetaminophen $4.2 \mathrm{~g}$ daily for pain control. Within 10 days, there was improvement with a marked decrease in pain and the patient was able to discontinue the acetaminophen. By 3 weeks, some of the ulcers were beginning to heal with a decrease in erythema and edema of the legs. At this point, the patient was able to ambulate without crutches. After completing 6 weeks of therapy without any side effects, the ulcers were completely healed and the patient elected to discontinue the medication. All the improvement occurred using sulfasalazine as monotherapy, without bed rest or the use of stockings. She returned 5 months later, off therapy, without any recurrence of the lesions (Fig. 1B).

\section{Case 2}

- A 33-year-old black woman presented in September 1988 with a 5-year history of tender, chronic ulcers of the lower aspects of the legs. There had been an increase in the number of ulcers and worsening pain over the previous 6 weeks, to the extent that she was now housebound and had to use a wheelchair. She had hypertension, which was controlled with hydralazine $25 \mathrm{mg}$ tid, 

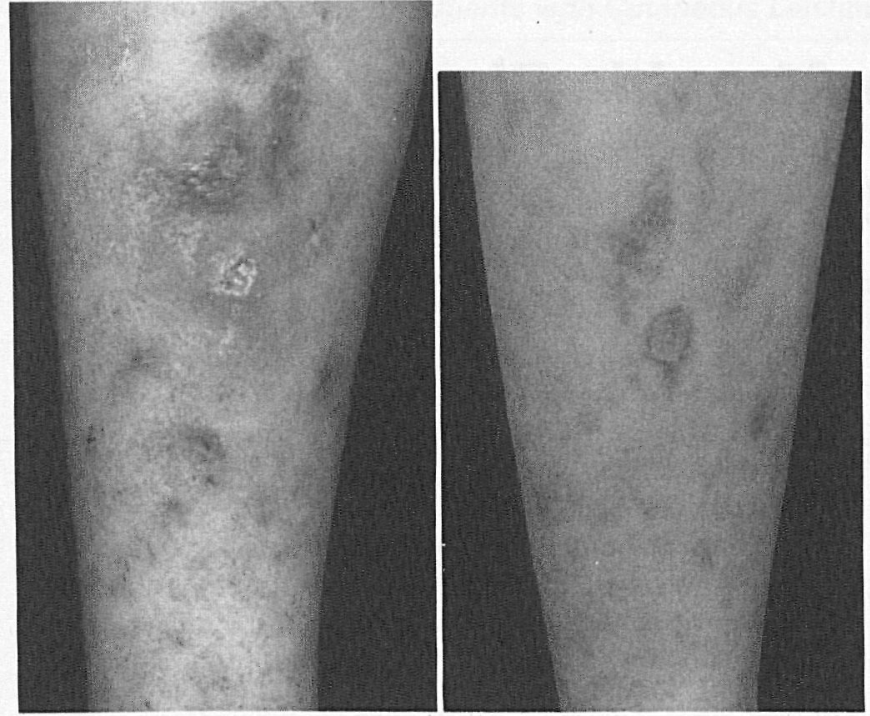

Figure 1. Case 1: atrophie blanche at $(\mathrm{A}$, left) pretherapy and $(\mathrm{B}$, right) 5 months following a 6 -week course of sulfasalazine $1 \mathrm{~g}$ three times a day.

verapamil hydrochloride SR $240 \mathrm{mg}$ bid, and hydrochlorthiazide $50 \mathrm{mg}$ daily. The patient was otherwise in good health, with no evidence of collagen vascular disease. Past treatments had been unsuccessful and included topical and oral corticosteroids, pentoxifylline, aspirin, oral erythromycin, and local dressings.

On examination, the clinical picture was similar to that described in case 1 , except that there were a greater number of white, atrophic scars (Figure 2A). Peripheral pulses were normal and there was no evidence of stasis dermatitis or varicose veins.

Laboratory investigations included CBC, SMA12, ANA, complement levels, cryoglobulins, urinalysis, anticardiolipin antibody and coagulation studies, which were all negative or within the normal range. A biopsy from an active ulcer site was consistent with atrophie blanche, with the salient findings being similar to those in case 1 .

The patient was commenced on sulfasalazine 500 $\mathrm{mg}$ tid for 3 days, followed by $1 \mathrm{~g}$ tid, which was well tolerated. As in the previous case, the only topical therapy was the daily use of dry dressings. For pain relief, the patient was using propoxyphene $130 \mathrm{mg}$ daily. Healing of the ulcers and a decrease in erythema and pain occurred after 6 weeks of therapy. The patient was now able to discontinue use of the wheelchair and propoxyphene. After 12 weeks of therapy, all the lesions had healed with no new ones forming over the last 7 weeks (Fig. 2B). At this point, we were able to discontinue sulfasalazine. Prior to the therapy and throughout the treatment course, the patient continued to preform numerous household tasks due to her responsibilities as a homemaker. She was never able to be on bed rest and did not use stockings; her only therapy was sulfasalazine.

\section{Discussion}

Therapies that have been reported for atrophie blanche (livedo vasculitis) include medical treatments such as bed rest, elastic stockings, ${ }^{9,10}$ silver nitrate cautery, ${ }^{9,10}$ topical, intralesional, and oral corticosteroids, ${ }^{5,7,10}$ antiplatelet therapy with aspirin and dipyridamole, ${ }^{10,11}$ fibrinolytics such as phenformin and ethylestrenol, ${ }^{9}$ warfarin ${ }^{12}$ and minidose heparin, ${ }^{13}$ nifedipine, ${ }^{14}$ nicotinic acid, ${ }^{15}$ anti-inflammatory agents, ${ }^{15}$ sulfapyridine ${ }^{2,15}$ and pentoxifylline. ${ }^{16,17}$ Surgical therapies include sympathectomy ${ }^{7,15}$ as well as excision followed by skin grafting. ${ }^{18}$ Both our patients previously failed therapy with some of the above modalities, underlining the fact that none of the present therapies are completely satisfactory.

The two cases we have presented were substantially physically incapacitated prior to starting sulfasal-
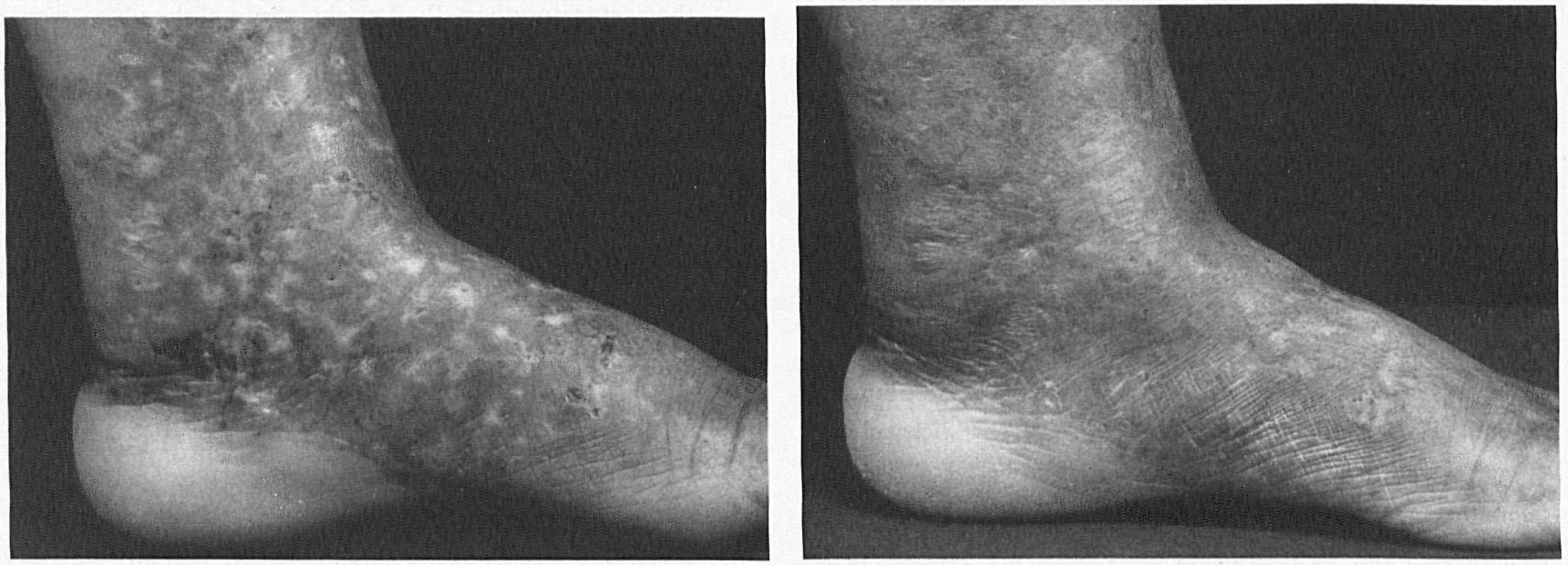

Figure 2. Case 2: atrophie blanche at (A, left) pretherapy and (B, right) 3 months following a 12-week course of sulfasalazine $1 \mathrm{~g}$ three times a day. 
azine. A clinical response with sulfasalazine therapy was first noted within 3 weeks in both cases, with complete healing at 6 weeks in one case and 12 weeks in the other. The mechanism of action of sulfasalazine in atrophie blanche is not clear. The active moiety may be the parent drug itself, its metabolites, or a combination of the two. Approximately $10-15 \%$ of sulfasalazine is absorbed in the small bowel. ${ }^{19}$ The remainder is metabolized in the large bowel to 5-aminosalicylic acid and sulfapyridine; 5-aminosalicylic acid (5-ASA) may reduce formation of cycloxygenase products in human colonic mucosa, including thromboxane $\mathrm{A}_{2},{ }^{20,21} \mathrm{a}$ stimulator of platelet aggregation. Whether these effects also occur in dermal vessels is not known. In addition, the other moiety, sulfapyridine, has been previously reported to be beneficial in some patients with atrophie blanche, ${ }^{2,15}$ possibly by acting as an antiinflammatory agent through its effects on polymorphonuclear leukocyte function ${ }^{22}$ and as a thromboxane inhibitor at high concentrations. ${ }^{23}$ Furthermore, the parent compound can also inhibit thromboxane synthetase in platelets $\mathrm{s}^{20,21}$ and partially inhibit cyclooxygenase. ${ }^{21}$ Another possibility is that sulfasalazine may prevent cytokine release from human mononuclear cells, ${ }^{24}$ and thereby inhibit platelet aggregation. ${ }^{25,26}$ For these reasons, sulfasalazine may be a preferred treatment over monotherapy with sulfapyridine or the nonsteroidal anti-inflammatory drugs. Further studies are necessary to understand the mechanism of action of sulfasalazine in this disease.

Atrophie blanche may have spontaneous remissions and exacerbations. Although possible, we think it is extremely unlikely that the improvement first seen in our patients within 3 weeks of starting sulfasalazine therapy was due to spontaneous disease fluctuation.

Sulfasalazine is a relatively inexpensive drug with a low incidence of severe side effects. Whatever the exact mechanism of action of sulfasalazine, in view of our preliminary positive results, further trials are warranted in atrophie blanche, a disease that can have a chronic debilitating course and one currently without consistently effective therapy.

\section{Acknowledgments}

The authors thank Amy J. Rundquist for typing the manuscript and Mark D. Young for taking the pictures.

\section{Drug Names}

\section{Sulfasalazine: Salazopyrin, Azulfidine}

\section{References}

1. Milian G. Les atrophies cutanees syphilitques. Bull Soc Fr Dermatol Syphilol. 1929;36:865.
2. Bard JW, Winkelmann RK. Livedo vasculitis: segmental hyalinizing vasculitis of the dermis. Arch Dermatol. 1967;96:489 499.

3. Schroeter AL, Diaz-Perez JL, Winkelmann RK, et al. Livedo vasculitis (the vasculitis of atrophie blanche): immunohistopathologic study. Arch Dermatol. 1975;111:188-193.

4. Stiefler RE, Bergfeld WF. Atrophie blanche. Int J Dermatol. 1982;21:1-7.

5. Milstone LM, Braverman IM, Lucky P, et al. Classification and therapy of atrophie blanche. Arch Dermatol. 1983;1 19:963-969.

6. Feldaker M, Hines EA, Kierland RR. Livedo reticularis with ulcerations. Circulation. 1956;23:196-216.

7. Gray HR, Graham JH, Johnson W, et al. Atrophie blanche: periodic painful ulcers of lower extremities. Arch Dermatol. 1966;93:187-193.

8. Shornick JK, Nicholes BK, Bergstresser PR, et al. Idiopathic atrophie blanche. J Am Acad Dermatol. 1983;8:792-798.

9. Gilliam JN, Herndon JH, Prystowsky SD. Fibrinolytic therapy for vasculitis of atrophie blanche. Arch Dermatol. 1974;109:664-667.

10. Drucker CR, Duncan WC. Antiplatelet therapy in atrophie blanche and livedo vasculitis. J Am Acad Dermatol. 1982;7:359-363.

11. Kern AB. Atrophie blanche. J Am Acad Dermatol. 1982;6:1048-1053.

12. Champion RH. Livedo reticularis with recurrent ulceration treated with anticoagulants. Br J Dermatol. 1962;74:195-196.

13. Jetton RL, Lazarus GS. Minidose heparin therapy for vasculitis of atrophie blanche. J Am Acad Dermatol. 1983;8:23-26.

14. Purcell SM. Nifedipine treatment of idiopathic atrophie blanche. J Am Acad Dermatol. 1986;14:851-854.

15. Winkelmann RK, Schroeter AL, Kierland RR, et al. Clinical studies of livedoid vasculitis (segmental hyalinizing vasculitis). Mayo Clin Proc. 1974;49:746-750.

16. Sams WM. Livedo vasculitis therapy with pentoxifylline. Arch Dermatol. 1988;124:684-687.

17. Sauer GC. Pentoxifylline (Trental) therapy for the vasculitis of atrophie blanche. Arch Dermatol. 1986;122:380-381.

18. Teman J, Ingber A, Rothem A, et al. Surgical treatment of livedo vasculitis. J Dermatol Surg Oncol. 1987;13:893-897.

19. Das KM, Sternlieb I. Salicylazosulfapyridine in inflammatory bowel disease. Dig Dis Sci. 1975;20:971-976.

20. Ligumsky M, Karmeli F, Sharon P, et al. Enhanced thromboxane $A_{2}$ and prostacyclin production by cultured rectal mucosa in ulcerative colitis and its inhibition by steroids and sulphasalazine. Gastroenterology. 1981;81:444-449.

21. Stenson WF, Lobos E. Inhibition of platelet thromboxane synthetase by sulfasalazine. Biochem Pharmacol. 1983;32:22052209.

22. Molin L, Stendahl O. The effect of sulfasalazine and its active components on human polymorphonuclear leukocyte function in relation to ulcerative colitis. Acta Med Scand. 1979;206:451457.

23. Hawkey CJ, Boughton-Smith NK, Whittle BJR. Modulation of human colonic arachidonic acid metabolism by sulfasalazine. Dig Dis Sci. 1985;30:1161-1165.

24. Cominelli F, Zipser RD, Dinarello CA. Sulfasalazine inhibits cytokine production in human mononuclear leukocytes: a novel anti-inflammatory mechanism. Clin Res. 1989;37:110A.

25. Coeugniet E, Bendixen G. Lymphokines and thrombosis. Acta Allergologica 1976;31:94-106.

26. Lavelle KJ, Ransdell BA, Trygstad CW. Identification of a new platelet aggregating factor released by sensitized leukocytes. Clin Immunol Immunopathol. 1975;3:492-502. 
This document is a scanned copy of a printed document. No warranty is given about the accuracy of the copy. Users should refer to the original published version of the material. 